\title{
Denoising of Seismic Signals Through Wavelet Transform Based on Entropy and Inter-scale Correlation Model
}

\author{
Zhi Cui*, Yixiang Wang \\ College of Information and Electronic Engineering, Hunan City University, Yiyang 413049, China
}

Corresponding Author Email: zhicui@yeah.net

https://doi.org/10.18280/i2m.180309

Received: 10 March 2019

Accepted: 1 June 2019

\section{Keywords:}

entropy, inter-scale correlation, seismic signal, denoising

\begin{abstract}
For effective removal of noises in seismic signals, this paper proposes an adaptive threshold denoising algorithm that integrates wavelet transform with entropy and inter-scale correlation (EIS) model. Firstly, noisy signals were decomposed by discrete wavelet transform, the highfrequency sub-bands on each scale were divided into equal subintervals, and the wavelet entropies of the subintervals were computed one by one. Secondly, the correlation coefficients of sampling points on each scale were calculated, and then compared with the high-frequency coefficients at corresponding positions. The comparison results, coupled with the wavelet entropies, were determine the noise variance of high-frequency sub-bands on each scale. Finally, the signals were reconstructed from the above results according to the new threshold function and the self-adaptive threshold rule. The experimental results show that our method outperformed several popular denoising approaches in terms of signal-to-distortion ratio (SDR), signal-to-noise ratio (SNR) and mean squared error (MSE).
\end{abstract}

\section{INTRODUCTION}

It is a crucial task to recognize and trace effective seismic signals in seismic surveillance. However, seismic signals are practically affected by pneumatic pressure, surf pounding, bad earth of detector embedment apparatus, and the scattering effect of near-surface objects with irregular contours. Therefore, it has always been a topical issue to improve efficiency and precision of seismic surveillance in the field of seismic surveillance to denoise seismic signals [1,2].

Filtering methods have long been applied by researchers in seismic signal denoising in spatial domain. Specifically, signals and noise are separated from each other according to different sorting between effective signals and noisy signals in spatial domain, such as median filtering, average filtering and Wiener filtering [3]. However, as a non-stationary process in essence, seismic shock does not fully accord with the theoretical foundation that traditional space-domain filtering methods rely on. Thus, the above filtering methods tend to smooth away edges or lose sensitive characteristics [4].

The theory of total variation, together with the deriving Rudin-Osher-Fatemi total variation, has become an important denoising method [5]. Total variation denoising has embraced good development due to its merit to retain the information of signal boundary. However, it is easy to trigger the staircase effect (Durand and Froment) [6, 7].

Another denoising method is the one based on transform domain. Specifically, Fourier transform, etc. is first used to project signals into transform domain through transform algorithm. Next, based on different distribution characteristics between effective signals and interference signals in transform domain, transformation coefficient that represents noise is eliminated by using feature distribution of transformation coefficient. Finally, inverse transformation is used to filter noise [8]. The basic recognition for the establishment of transform domain is that useful information in signals usually concentrates on the low-frequency range while noisy signals concentrate on the high-frequency range. Thus, based on frequency, effective separation of signals and noise can be realized.

Wavelet transform is one of the methods in transform domain. Once a random group of signals are decomposed by wavelet, the form of effective information, and noise is significantly different in wavelet domain. Thus, it appears that wavelet transform has overwhelmingly strong vitality and gains substantial development in the field of signal processing [9]. The emerging thresholding method is marked as an important development for wavelet transform. The method of wavelet domain denoising based on threshold processing, initialized by Dohono, can estimate the threshold value through wavelet transform coefficient and reconstruct signals with the retained wavelet coefficient. This method proves to reach appropriate optimum in the sense of least mean square errors, and endows good visual effects to denoised signals [10]. The key issue in wavelet threshold denoising lies in the design of threshold function. Traditional soft threshold will cause constant deviation between the estimated value and the actual value of wavelet coefficient, while the hard threshold, with its discontinuous function, will generate oscillation in reconstructed signals [11]. Given the above factors, researchers have proposed many improved schemes. For example, Dong and Yi put forward the eclectic non-smooth threshold value in [12], using a variable factor to adjust deviations in wavelet coefficient caused by soft threshold value. However, the threshold function like this was still discontinuous. Zhang et al. proposed a continuous threshold function in [13], which set evolving factors to enhance the denoising effect. Nevertheless, with empirical estimation instead of universal equation, the setting had some drawbacks. Shu proposed a threshold function with exponential factors in 
[14], with the exponential items' regulation to smooth function curves and reduce oscillation. But it was difficult to determine the value range of exponential items. Recently, Chen, Wang, et al proposed the denoising method based on wavelet entropie, which determined the threshold at various scales by calculating high-frequency wavelet entropies at various scales after signals were decomposed. However, high-frequency wavelet entropies at various scales like those are regarded as noise coefficient, excluding the factor that is part of useful information in the high-frequency zone. Thus, the denoising method based on wavelet entropie, once adopted, may cause loss of some details for denoised signals and even the risk of signal distortion.

This paper proposed an adaptive wavelet domain denoising method with the wavelet entropy-inter scale correlation model. On the basis of the method in [15], two improvements were proposed. The first was a proposed denoising model that combined wavelet entropie with correlation among scales. The proposed method was different from the aforementioned methods in $[12,14,15]$. Instead, high-frequency relative coefficient at various scales was calculated to filter out useful information in the high-frequency zone, and standard deviation of noise at various scales was determined according to wavelet entropies. The second improvement lied in a proposed new differentiable threshold function that controlled the attenuation of high-frequency coefficient self-adaptively. Whereas the soft threshold function continued its application in [15].

\section{RELATED WORK}

This section serves as an explanation of some basic knowledge.

\subsection{Theory of wavelet transformation}

Assuming that $\phi(t)$ was a square integrable function, and if Fourier transform $\phi(\omega)$ satisfied the requirement of [16].

$$
\int_{R} \frac{|\phi(\omega)|^{2}}{\omega} d \omega<\infty
$$

Then $\phi(t)$ was called as fundamental wavelet or mother wavelet. With the following transformation, the wavelet basis function was obtained:

$$
\varphi_{a, b}(t)=|a|^{-\frac{1}{2}} \varphi\left(\frac{t-b}{a}\right), \quad b \in R, a \in R-\{0\}
$$

where, $a$ denoted scalability factor, and $b$ denoted shift factor.

The discrete wavelet transform was defined as:

$$
W_{f}(a, b)=\int f(t) \phi_{a, b}^{*}(t) d t \quad(j=0,1,2 \cdots, k \in Z)
$$

And the discrete wavelet transforms coefficient of the signal $f(t)$ was:

$$
C_{j}^{k}=\int_{-\infty}^{+\infty} f(t) \bar{\phi}_{j, k}(t) d t
$$

\subsection{Theory of threshold denoising method}

Under normal conditions, the seismic signals received by instrument ends could be approximately represented by the following equation:

$$
h(i)=c(i)+d(i) \quad(i=1,2, \cdots N)
$$

where, $c(i)$ denoted ideal signal, $d(i)$ denoted doping noise, and $h(i)$ denoted the signals that were polluted by noise.

Wavelet transform was used for the signal $h(i)$ in the following expression:

$$
W T_{j, k}=\int h(i) \phi_{j, k}(i) d i=\int c(i) \phi_{j, k}(i) d i+\int d(i) \phi_{j, k}(i) d i
$$

where, $\phi_{j, k}$ denoted discrete wavelet.

Equation (5) could be rewritten as:

$$
\omega_{j, k}=\mu_{j, k}+v_{j, k}
$$

where, $\omega_{j, k}$ denoted wavelet coefficient with noised signals, $\mu_{j, k}$ and $v_{j, k}$ denoted wavelet coefficient for effective information and that for noise, respectively.

\section{THE DE-NOISING METHOD USING EIS MODEL}

In this section, the proposed method is described in detail below. Here was two key points: for the first one, by calculating the high-frequency coefficient of the wavelet entropy at various scales, the maximum distribution section would be preliminarily determined with the maximum noise energy. Then, it was decided whether the above section was the noise-dominant one through the comparison of the sum of wavelet coefficient with relative coefficient. For the second point, a new derivable threshold function was designed, which helped reduce the constant deviation of wavelet coefficient resulted from discontinuous threshold function.

\subsection{The new threshold function}

The methods of wavelet threshold denoising was divided into the hard threshold method and the soft threshold method. The difference was small between $\omega_{j, k}$ of the ideal signal (without noise) that was estimated by the hard threshold method and $\bar{\omega}_{j, k}$ of the actual signal. However, the discontinuity of $\omega_{j, k}$ at the threshold $\lambda$ caused the estimated signal to generate additional oscillation at the singular point of the signal, thus the denoised signal failed to have the smooth boundary as that for the ideal signal. The wavelet coefficient that was obtained by the soft threshold method had a good global continuity. However, the constant derivation between $\omega_{j, k}$ of the ideal signal that was estimated by the hard threshold method and $\bar{\omega}_{j, k}$ of the actual signal caused a low approximating level between the denoised signal and the ideal signal, and affected the precision of signal reconstruction $[17$, 18]. There were two characteristics for a good threshold function: first, it would render the deviation between the estimated value and the actual value of the wavelet coefficient to be as small as possible; second, it was continuous in the wavelet domain. 
The new threshold function was established as follows:

$$
\bar{\omega}_{j, k}=\left\{\begin{array}{c}
\operatorname{sign}\left(\omega_{j, k}\right) \cdot \frac{1}{e^{\left|\omega_{j, k}\right|-\lambda_{j}}} \cdot \sqrt{\frac{\omega_{j, k}^{2}+\left(\left|\omega_{j, k}\right|-\lambda_{j}\right)^{2}}{2}},\left|\omega_{j, k}\right| \geq \lambda_{j} \\
0,\left|\omega_{j, k}\right|<\lambda_{j}
\end{array}\right.
$$

There was constant deviation between the absolute value of $\bar{\omega}_{j, k}$ that was obtained totally by the soft threshold method and $\omega_{j, k}$. Such deviation should be reduced by every means. However, if $\lambda_{j}$ was equal to 0 , the value of $\bar{\omega}_{j, k}$ was the same as the value that was obtained by the hard threshold method. From equation (6) it could be seen that there was $\mu_{j, k}$ (represented effective information) and $v_{j, k}$ (represented noise) in $\omega_{j, k}$. To obtain the minimum value of $\left\|\omega_{j, k}-\mu_{j, k}\right\|$ within the norm meaning of $l_{2}$ was the intention of signal construction. From observation of equation (7), it could be seen that $\sqrt{\frac{\omega_{j, k}^{2}+\left(\left|\omega_{j, k}\right|-\lambda_{j}\right)^{2}}{2}}$ was the geometric average between $\omega_{j, k}$ and $\left|\omega_{j, k}\right|-\lambda_{j}$, and $\frac{1}{e^{\left|\omega_{j, k}\right|-\lambda_{j}}}$ was a regulatory item. If $\left|\omega_{j, k}\right| \geq \lambda_{j} \quad, \quad$ as $\quad\left|\omega_{j, k}\right| \quad$ increased, $\frac{1}{e^{\left|\omega_{j, k}\right|-\lambda_{j}}}$ decreased continuously, working to dynamically regulate threshold. The new threshold function could determine the attenuation of wavelet coefficient self-adaptively, thus reducing lass of useful information in the high-frequency zone and improving the signal-to-noise ratio of the reconstructed signals.

\subsection{Calculation of wavelet entropy}

According to the information theory, entropy denoted the average information amount for each symbol in information sources, and also denoted the average uncertainty of information sources. In other words, the uniformity of probability distribution of the signal was decided by entropy.

From the viewpoint of energy conservation of wavelet transform, after discrete wavelet transform was used for $h(i)$, the high-frequency coefficient at the $k$ th position under the decomposition scale of $j(j=1,2, \cdots L)$ was recorded as $d_{j, k}$, and the wavelet energy under $j$ could be expressed as:

$$
E_{j}=\sum_{k}\left|d_{j, k}\right|^{2} \quad(k=1,2, \cdots M)
$$

Thus, the total sum of energy under all of the decomposition scales was:

$$
E=\|h(i)\|^{2}=\sum_{j} \sum_{k}\left|d_{j, k}\right|^{2}=\sum_{j} E_{j}
$$

Assuming that the number of sampling points for the section of signals was $N$. if the high-frequency coefficient at the $j$-th scale was divided into $n$ subsections, the wavelet energy at the $m$-th subsection could be expressed as:

$$
E_{j}(m)=\sum_{k}^{N / n}\left|d_{j, k}\right|^{2}
$$

where, $\frac{N}{n}$ denoted the number of sampling points that were contained in each subsection.

The probability density of the wavelet energy at the $m$ th subsection against the total energy at its scales was then calculated as:

$$
p_{m}=\frac{E_{j}(m)}{E_{j}}
$$

Finally, the wavelet entropy (represented by $S_{m}$ ) of the $m$ th subsection was calculated as:

$$
S_{m}=-\sum_{j} p_{m} \ln p_{m}
$$

According to the above analysis, as the combination of wavelet transform and the information entropy, the wavelet entropy could quantitatively describe the characteristics of energy distribution on the time-frequency domain. The definition of wavelet entropy was introduced to calculate the noise energy of high-frequency wavelet coefficient at various scales. In this way, the noise dominant section at each scale would be judged effectively so that the noise level at each scale would be determined.

\subsection{Inter-scale correlation}

Research results have shown that after a specific signal went through wavelet transform, the wavelet coefficient that represented useful information was strongly related to each other among different scales, but the wavelet coefficient that represented noise were not characterized by such character [19]. The relative coefficient among different scales was used to measure such correlative characters, which was defined as [20]:

$$
\operatorname{Cor}_{j, k}(j)=d_{j, k}(j) \cdot d_{j+1, k}(j+1)
$$

where, $\operatorname{Cor}_{j, k}(j)$ denoted the relative coefficient at the $k$-th position at the $j$-th scale, $d_{j, k}(j)$ and $d_{j, k}(j)$ denoted the highfrequency coefficient at the $k$-th position at the $j$ th scale and at the $(j+1)$-th scale, respectively.

To facilitate calculation and comparison, the paper performed normalization processing on equation (13) as:

$$
\operatorname{NCor}_{j, k}(j)=\operatorname{Cor}_{j, k}(j) \cdot \sqrt{\sum_{k=1}^{M}\left|\omega_{j, k}(k)\right|^{2} / \sum_{k=1}^{M}\left|\operatorname{Cor}_{j, k}(k)\right|^{2}}
$$

where, $\mathrm{NCor}_{j, k}(j)$ denoted the relative coefficient for normalization, and each item in the radical sign denoted the calculation energy.

As the magnitude calculation to normalize relative coefficient was complex, the paper proposed a new relative correlation $\mathrm{New}-\mathrm{Cor}_{j, k}(j)$ to streamline it. $\mathrm{Max}-d_{j, k}$ was defined to denote the maximum value of high-frequency coefficient at the $k$-th position at the $j$-th scale, and $M i n-d_{j, k}$ denoted the minimum value as such. The new normalized correlation was expressed as:

$$
N e w-\operatorname{Cor}_{j, k}(j)=\frac{1}{a} \cdot \sqrt{\frac{\left(\operatorname{Max}-d_{j, k}(j)\right)^{2}+\left(\operatorname{Min}-d_{j, k}(j)\right)^{2}}{2}}
$$

where, $a$ denoted regulatory factors. When the value of $N e w-\operatorname{Cor}_{j, k}(j)$ exceeded 1 , the wavelet coefficient at various scales shared strong correlation, which represented where useful information was located; when the value of New $-\operatorname{Cor}_{j, k}(j)$ is small than 1 , the wavelet coefficient at 
various scales shared weak correlation, which represented where noise was located.

\subsection{Adaptive threshold rule}

In wavelet threshold denoising, it was of great importance to define threshold rules that directly influence the denoising effects of signals. Chitsaz et al. proposed a threshold rule as follows [21]:

$$
T=\sigma \sqrt{\frac{2 \ln N}{\ln (j+1)}}
$$

where, $T$ denoted the threshold value, $N$ denoted the signal length, $j$ denoted levels of decomposition, and $\sigma$ denoted the standard deviation of noise, whose value was:

$$
\sigma=\frac{\operatorname{median}\left(\left|d_{j, k}\right|\right)}{0.6745}
$$

By combining the works in [22], this paper improved the self-adaptability of the threshold rules in equation (17). The high-frequency subsection of signals through wavelet decomposition was recorded as $H_{j}$. The wavelet coefficient at the $k$-th position in the subsection was recorded as $d_{j, k}$. The paper also defined a neighborhood window (represented by $W$ ) whose center lied in the position of $d_{j, k}$ and whose width was $M$. Thus, the variance of $h(i)$ in $W$ at $H_{j}$ could be expressed as:

$$
\sigma_{h}=\sqrt{\frac{\sum\left|d_{j, k}\right|^{2}}{M}}
$$

According to the aforementioned analysis, there was also useful information in the high-frequency zone that was decomposed by signals, thus the value of $\left|\sigma_{h}-\sigma\right|$ was not equal to 0 . $\left|\sigma_{h}-\sigma\right|$ was recorded as $\sigma_{s}$, denoting the variance of useful information in $W$. The improved threshold rule was expressed as follows:

$$
T_{\text {new }}=\sqrt{\frac{\sigma^{2}+\sigma_{s}^{2}}{2}} \cdot T
$$

\subsection{The proposed method}

On the basis of the above analysis, the procedure of algorithm herein was summarized as follows:

(1) Determined the appropriate wavelet basis and the scale of decomposition, and applied discrete wavelet transform to noised seismic signals until the high-frequency coefficient and low-frequency coefficient at various scales were obtained. Among them, the wavelet basis was determined by comparison, and the decomposition scale was determined on the basis of the methods proposed in [23].

(2) High-frequency wavelet coefficient at various scale were divided equally into $n$ subsections. The wavelet entropy in each subsection was calculated by equation (13) until the subsection with maximum wavelet entropy was found out and was marked as $m$.

(3) The correlation amounts among different scales for the high-frequency wavelet coefficient were calculated by equation (14) and equation (16). The sum of correlation amounts for each subsection and the sum of wavelet coefficient was compared in size.

(4) Decide whether the sum of wavelet coefficient of $m$ in step (2) exceeded that of correlation amounts. If so, $m$ was then the noise-dominant section at this scale. The noise variant in $m$ was calculated as that of the scale.

(5) In step (4), if not, the correlation amounts of highfrequency coefficient at various scales that were calculated in step (3) was compared with the wavelet coefficient at each sampling point, until the points were found whose correlation amounts were smaller than the wavelet coefficient. Maintained the wavelet coefficient of the points, which denoted noise and was recorded as $d_{j, k}^{\prime}$, and abandoned that of the rest points.

(6) On the premise of keeping the number of sampling points unchanged, the positions of $d_{j, k}^{\prime}$ were fixed to constitute the high-frequency wavelet coefficient at various scales. Then, according to the method in step (2), divided the highfrequency coefficient at various scales into $n$ subsections whose average value of coefficients was calculated one by one. The subsection with the largest average value was determined as the noise-dominant section. The noise variant at the present scale was decided by the subsection with the largest average value.

(7) Calculated the threshold value $T_{\text {new }}$ at various scales according to equation (20). Equation (8) was used to handle the high-frequency coefficient that was obtained in step (6) so that a new high-frequency coefficient was acquired.

(8) The low-frequency coefficient of the highest decomposition level and the new high-frequency coefficient that was acquired in step (7) were together used to reconstruct denoised signals.

\section{EXPERIMENTS}

In this section, three different methods were introduced for comparison to detect the performance of the method herein. The parameters of the proposed method are set as: $\mathrm{a}=0.65$ and $\mathrm{M}=5$. The parameters of the compared methods were set as default to achieve optimal results. SDR (Signal to Distortion Ratio), SNR (Signal to Noise Ratio) and MSE (Mean Square Error) herein were used as objective indices to evaluate the quality of denoised signals. All the experiments were implemented on a Core i5(R) $3.2 \mathrm{GHz}$ PC with 8 GB RAM.

In seismic exploration, seismic signals that were received by the receiving end were the convolution of seismic wavelet and reflection coefficient. Studying seismic wavelet played an important role in implementing precise research on the rule of seismic wave transmission. The energy-concentrated Ricker wave, $\mathrm{P}$ wave and $\mathrm{S}$ wave were commonly used in seismic analysis as simulative signals, which would solve problems of reflection together with reflection function convolution. Thus, in the first experiment, we aim at the above three signals. The SDR results herein were compared with those of hard threshold method (HT), soft threshold method (ST), and the method in [18]. Figure 1 showed the comparison results.

The SDR results in all the three images of Figure 1 showed that the proposed method outperformed the other methods. For example, with the input SNR of 0 of the Ricker signal, the SDR result obtained by the proposed method is 0.0996 , with the decrease value of $0.0407,0.0283$ and 0.0186 respectively over the values obtained by the HT, ST and the method in [18]. Also, the results obtained by the proposed method outmatched the others for different signals with different input SNR, with the 
average rise at $0.040,0.022$ and 0.011 on average, respective to in HT, ST and the method in [18]. It means when de-noised by the compared methods, some important details in the signals would disappear together with the noise. The proposed method helped effectively maintain structure characteristics and retain better useful information in signals.

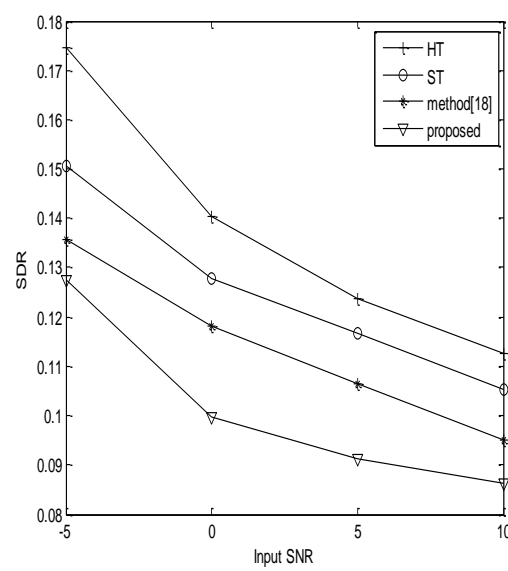

(a) Results on Ricker wave

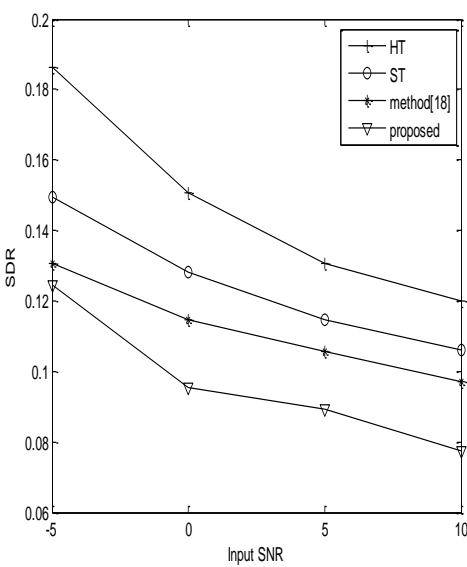

(b) Results on P wave

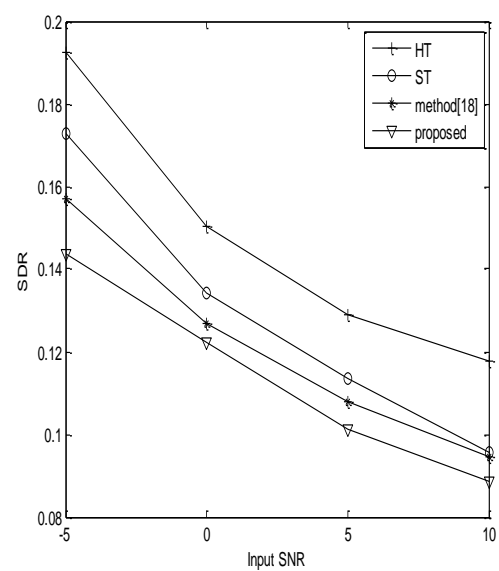

(c) Results on S wave

Figure 1. The SDR results obtained by four compared methods on three test signals

The SNR and MSE results were compared with those of the other three methods in the second experiment that was performed on the same signals: "Ricker", "P wave" and "S wave". The comparison results are shown in Tab. 1, Tab. 2 and Tab. 3. It can be found that the results obtained by the proposed method are better than the other methods for the same signal with the same input SNR. For instance, with the input SNR of 5 of "P wave", the output SNR result obtained by the proposed method is $14.766 \mathrm{~dB}$, increasing by $5.637 \mathrm{~dB}, 2.242 \mathrm{~dB}$ and $0.95 \mathrm{~dB}$ respectively compared with the values in HT, ST and the method in [18]. We also found that the results obtained by the proposed method are better than the others for different signal with different input SNR. For instance, the output SNR result obtained by the proposed method is increased by 4.393 $\mathrm{dB}, 1.675 \mathrm{~dB}$ and $0.710 \mathrm{~dB}$ on average, respective to HT, ST and the method in [18]. In the meantime, the MSE result obtained by the proposed method is decreased by $0.205,0.118$ and 0.041 on average, respective to HT, ST and the method in [18].

As a final experiment, we compared the de-noised effects achieved by the proposed method, HT, ST and the method in [18]. Figure 2 offers the visual comparison between them. From the visual results, we can see that the proposed method can get better results than the other methods.

Table 1. Performance of the de-noised methods of Ricker wave by Output SNR (dB) and MSE

\begin{tabular}{ccccccccc}
\hline \multirow{2}{*}{ Input SNR (dB) } & \multicolumn{4}{c}{ Output SNR (dB) } & \multicolumn{4}{c}{ MSE } \\
\cline { 2 - 8 } & HT & ST & Method [18] & Proposed & HT & ST & Method [18] & Proposed \\
0 & 4.058 & 6.303 & 7.429 & 8.051 & 0.671 & 0.485 & 0.368 & 0.302 \\
5 & 9.832 & 10.992 & 11.246 & 11.935 & 0.426 & 0.366 & 0.306 & 0.273 \\
10 & 12.689 & 14.431 & 15.147 & 16.059 & 0.372 & 0.319 & 0.298 & 0.254 \\
\hline
\end{tabular}

Table 2. Performance of the de-noised methods of $P$ wave by Output SNR (dB) and MSE

\begin{tabular}{ccccccccc}
\hline \multirow{2}{*}{ Input SNR (dB) } & \multicolumn{4}{c}{ Output SNR (dB) } & \multicolumn{4}{c}{ MSE } \\
\cline { 2 - 9 } & HT & ST & Method [18] & Proposed & HT & ST & Method [18] & Proposed \\
0 & 5.814 & 8.299 & 9.084 & 9.907 & 0.601 & 0.493 & 0.357 & 0.329 \\
5 & 9.129 & 12.524 & 13.816 & 14.766 & 0.447 & 0.384 & 0.319 & 0.301 \\
10 & 13.354 & 15.063 & 16.402 & 16.978 & 0.325 & 0.311 & 0.294 & 0.239 \\
\hline
\end{tabular}

Table 3. Performance of the de-noised methods of S wave by Output SNR (dB) and MSE

\begin{tabular}{ccccccccc}
\hline \multirow{2}{*}{ Input SNR (dB) } & \multicolumn{4}{c}{ Output SNR (dB) } & \multicolumn{3}{c}{ MSE } \\
\cline { 2 - 9 } & HT & ST & Method [18] & Proposed & HT & ST & Method [18] & Proposed \\
0 & 5.793 & 8.908 & 10.114 & 10.783 & 0.718 & 0.613 & 0.421 & 0.343 \\
5 & 8.366 & 13.429 & 14.741 & 15.626 & 0.475 & 0.352 & 0.314 & 0.295 \\
10 & 12.742 & 16.288 & 16.957 & 17.213 & 0.394 & 0.328 & 0.286 & 0.256 \\
\hline
\end{tabular}




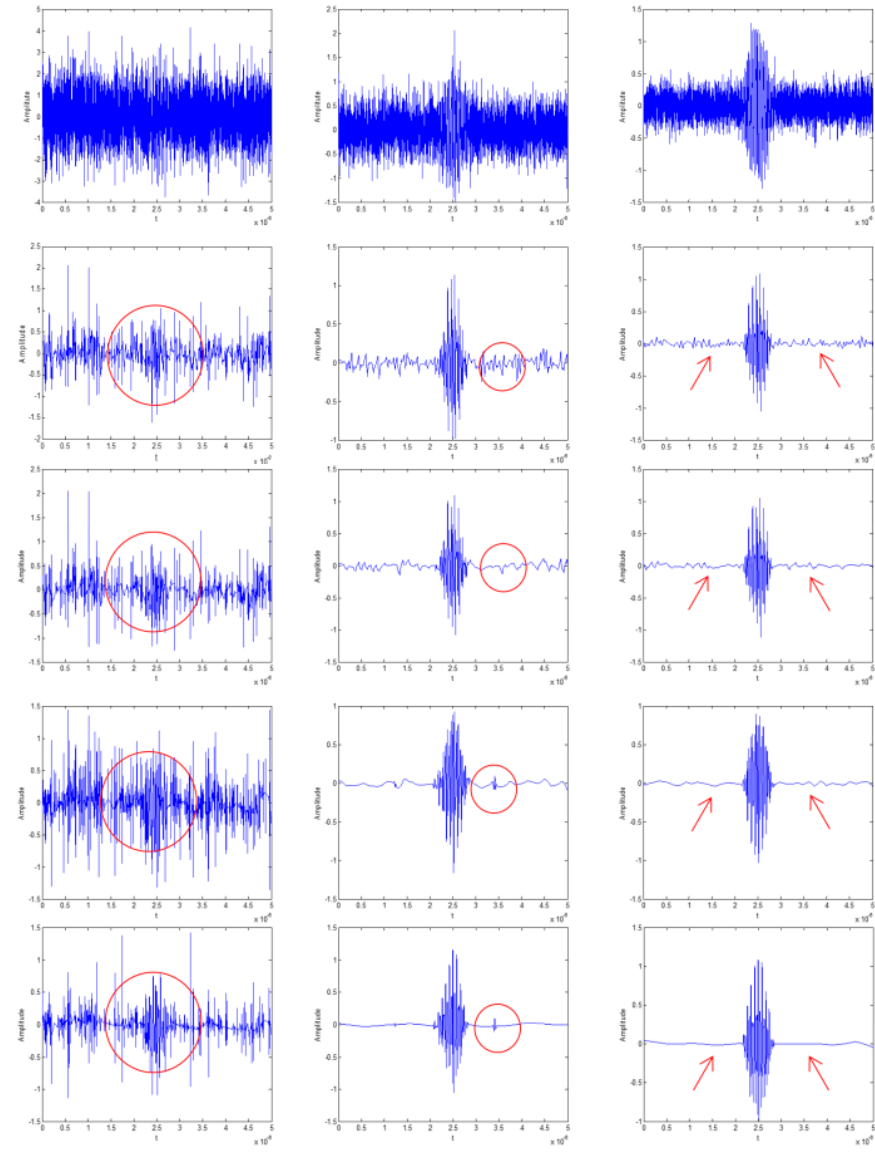

Figure 2. Visual comparison of the de-noising results. The first row: testing signal, with $\sigma=-15,-5$ and 0 . The second row: de-noising results by using HT. The third row: denoising results by using ST. The fourth row: de-noising results by using method in [18]. The final row: de-noising results obtained by the proposed method

\section{CONCLUSIONS}

This paper proposed an improved EIS Model to denoise seismic signals, whose denoising effects were better than those of other mentioned methods. First, through discrete wavelet transform, seismic signals were decomposed into the lowfrequency part and the high-frequency part, following the division of high-frequency subbands of various decomposed scales into several subsections. Then, wavelet entropies of each subsection were calculated, their sizes being referred to in preliminary calibration of noise-dominant sections at various scales that was done to determine noise variants at various scales and to facilitate threshold denoising. Next, the paper proposed an improved equation of correlation magnitude to calculate correlation coefficient for each sampling points at various scales. Based on correlation coefficient and the size of wavelet coefficient, the maximum energy section of noise was decided, and the noise coefficient were calculated to the maximum precision. When the noise variants at various scales were calculated, the proposed new threshold function and the self-adaptability threshold rules were used to process the noise coefficient. Experiments showed that the proposed method helped obtain better SNR data and SDR data. With less abrupt denoised signals and better smoothness, the proposed method facilitated seismic researchers to draw accurate judgments. However, as it was required for the method to calculate correlated magnitude and conduct point-to-point comparison with wavelet coefficient, and as the maximum section of wavelet entropies should be combined with the comparison results before further calculation was done, the efficiency of the proposed method was weakened. In future work, the researchers herein will consider a method with higher sparsity adaptive signals.

\section{ACKNOWLEDGMENTS}

This work is supported by Yiyang Science and Technology Bureau under Grants number YKZ [2016] 51 \& 2017YC01. The authors would like to thank the anonymous reviewers for their insightful comments and suggestions, which have greatly improved this paper.

\section{REFERENCES}

[1] Tian, Y., Li, Y. (2014). Parabolic-trace time-frequency peak filtering for seismic random noise attenuation. IEEE Transactions on Geoscience and Remote Sensing, 11(1): 158-162. https://doi.org/10.1109/LGRS.2013.2250906

[2] Karthikeya, P., Murugappan, M., Yaacob, S. (2012). ECG signal denoising using wavelet thresholding techniques in human stress assessment. International Journal on Electrical Engineering and Informatics, 4(2): 306-317. https://doi.org/10.15676/ijeei.2012.4.2.9

[3] Iordache, M.D., Bioucas-Dias, J.M., Plaza, A. (2012). Total variation spatial regularization for sparse hyperspectral unmixing. IEEE Transactions on Geoscience and Remote Sensing, 50(11): 4484-4502. https://doi.org/10.1109/TGRS.2012.2191590

[4] Li, C., Yin, W., Jiang, H. (2013). An efficient augmented Lagrangian method with applications to total variation minimization. Computational Optimization and Applications, 56(3): 507-530. https://doi.org/10.1007/s10589-013-9576-1.

[5] Vogel, C.R., Oman, M.E. (1996). Iterative methods for total variation denoising. SIAM Journal on Scientific Computing, $\quad 17$ : https://doi.org/10.1137/0917016

[6] Ma, J., Fenn, M. (2006). Combined complex ridgelet shrinkage and total variation minimization. SIAM Journal on Scientific Computing, 28(3): 984-1000. https://doi.org/10.1137/05062737X

[7] Singh, B.N., Tiwari, A.K. (2006). Optimal selection of wavelet basis function applied to ECG signal denoising. Digital Signal Processing, 16(3): 275-287. https://doi.org/10.1016/j.dsp.2005.12.003

[8] Alfaouri, M., Daqrouq, K. (2008). ECG signal denoising by wavelet transform thresholding. American Journal of Applied Sciences, 5(3): 276-281. https://doi.org/10.3844/ajassp.2008.276.281

[9] Wang, Y., He, Z., Zi, Y. (2010). Enhancement of signal denoising and multiple fault signatures detecting in rotating machinery using dual-tree complex wavelet transform. Mechanical Systems and Signal Processing, 24(1):

119-137. https://doi.org/10.1016/j.ymssp.2009.06.015

[10] Zhang, Q., Liu, H., Zhou, X. (2006). Wavelet softthreshold denoising method of power quality signal. High Voltage Engineering, 32(1): 99-101. https://doi.org/10.3969/j.issn.1003-6520.2006.01.037 
[11] Dong, Y., Yi, X. (2006). Wavelet denoising based on four improved functions for threshold estimations. Journal of Mathematics, 11: 158-162. https://doi.org/10.3969/j.issn.1003-6520.2006.01.037

[12] Zhang, Q., Liu, H., Lan, Q. (2008). Power quality signal denoising based on wavelet hybrid threshold method. Electric Power Automation Equipment, 28(8): 28-30. https://doi.org/10.3969/j.issn.1006-6047.2008.08.006

[13] Chen, Y., Li, S. (2006). Application of improved threshold denoising based on wavelet transform to ultrasonic signal processing. Journal of Beijing University of Aeronautics and Astronautics, (4): 158-162. https://doi.org/10.3969/j.issn.1001-5965.2006.04.021

[14] Ren, W.X., Sun, Z.S. (2014). Structural damage identification by using wavelet entropy. Engineering $\begin{array}{lll}\text { Structures, } & 30(10) \text { : } & \text { 2840-2849. }\end{array}$ https://doi.org/10.1016/j.engstruct.2008.03.013

[15] Lin, J., Qu, L. (2000). Feature extraction based on Morlet wavelet and its application for mechanical fault diagnosis. Journal of Sound and Vibration, 234(1): 158-162. https://doi.org/10.1006/jsvi.2000.2864

[16] Selesnick, I.W., Baraniuk, R.G., Kingsbury, N.G. (2005). The dual-tree complex wavelet transform. IEEE Signal Processing Magazine, 22(6): 123-151. https://doi.org/10.1109/MSP.2005.1550194

[17] Donoho, M.N., Vetterli, M. (2014). Wavelet-based texture retrieval using generalized Gaussian density and Kullback-Leibler distance. IEEE Transactions on Image Processing, 11(2): 146-158. https://doi.org/10.1109/83.982822
[18] Chen, X.J., Wang, W.T., Jia, M.C., Song, N. (2014). New denoising method for speech signal based on wavelet entropy and adaptive threshold. Application Research of Computers, 31(3): 753-755. https://doi.org/10.3969/j.issn.1001-3695.2014.03.027

[19] Duan, Y., Ma, L., Li, Y.J., Wang, T.H. (2010). Improved soft-threshold denoising algorithm based on wavelet analysis. Science Technology and Engineering, 10(23): 5755-5758. https://doi.org/10.3969/j.issn.16711815.2010.23.035

[20] Gao, G.R., Liu, Y.P., Pan, Q. (2012). A differentiable thresholding function and an adaptive threshold selection technique for pulsar signal denoising. Acta Physica Sinica, 61(13): 139701. https://doi.org/10.7498/aps.61.139701

[21] Chitsaz, H., Amjady, N., Zareipour, H. (2015). Wind power forecast using wavelet neural network trained by improved Clonal selection algorithm. Energy Conversion and Management, 89(1): 588-598. https://doi.org/10.1016/j.enconman.2014.10.001

[22] Cui, Z., Cui, X.P. (2016). Ultrasonic signal de-noising based on wavelet entropy and inter-scale correlation. International Journal of Multimedia and Ubiquitous Engineering, 11(1): 135-146. https://doi.org/10.14257/ijmue.2016.11.1.14

[23] Feng, Z.H., Miao, C.Y., Bai, H. (2016). De-noising algorithm of ultrasonic echo signal based on wavelet transform and independent component analysis. International Journal of Control and Automation, 9(11): 375-384. https://doi.org/10.14257/ijca.2016.9.32 